\title{
Brief Report: Normal Intestinal Permeability at Elevated Platelet Serotonin Levels in a Subgroup of Children with Pervasive Developmental Disorders in Curaçao (The Netherlands Antilles)
}

\author{
Ramses F. J. Kemperman · Fred D. Muskiet • \\ A. Inge Boutier · Ido P. Kema - Frits A. J. Muskiet
}

Published online: 28 July 2007

(C) Springer Science+Business Media, LLC 2007

\begin{abstract}
This study investigated the relationship between platelet (PLT) serotonin (5-HT) and intestinal permeability in children with pervasive developmental disorders (PDD). Differential sugar absorption and PLT 5-HT were determined in 23 children with PDD. PLT 5-HT (2.0-7.1 nmol/ $10^{9}$ PLT) was elevated in $4 / 23$ patients. None exhibited elevated intestinal permeability (lactulose/mannitol ratio: $0.008-0.035 \mathrm{~mol} / \mathrm{mol})$. PLT $5-\mathrm{HT}$ did not correlate with intestinal permeability or GI tract complaints. PLT 5-HT correlated with $24 \mathrm{~h}$ urinary 5-hydroxyindoleacetic acid (5-HIAA; $p=.034)$. Also urinary 5-HIAA and urinary 5 -HT were interrelated $(p=.005)$. A link between hyperserotonemia and increased intestinal permeability remained unsupported. Increased PLT 5-HT in PDD is likely to derive from increased PLT exposure to 5-HT. Longitudinal studies, showing the (in)consistency of abnormal intestinal permeability and PLT 5-HT, may resolve present discrepancies in the literature.
\end{abstract}

Keywords Child development disorders - Pervasive . Platelets · Serotonin · Gastrointestinal · Permeability

R. F. J. Kemperman $(\bowtie) \cdot$ I. P. Kema •

F. A. J. Muskiet

Department of Pathology and Laboratory Medicine,

CMC-V 1st Floor, Room Y3.181, University Medical Center

Groningen, P.O. Box 30.001, 9700 RB Groningen,

The Netherlands

e-mail: r.f.j.kemperman@rug.nl

F. D. Muskiet

Department of Pediatrics, St. Elisabeth Hospital, Curaçao,

The Netherlands Antilles

A. I. Boutier

Sentro Inge Boutier, Practice for Child Therapy, Curaçao,

The Netherlands Antilles

\section{Introduction}

Autism has been linked to gastrointestinal (GI) disturbances (White 2003). It is, however, questionable whether GI anomalies in children with autism are specific (Erickson et al., 2005). An increase of chronic diarrhea, constipation, abdominal bloating and food regurgitation has been found in some studies, but could not be confirmed in more recent studies (reviewed by Erickson et al. 2005). Increased GI permeability, as established through the differential sugar absorption test (SAT), was demonstrated in 9/21 (43\%) (D'Eufemia et al., 1996) and 19/26 (76\%) (Horvath \& Perman, 2002) of patients diagnosed with autism. The SAT measures the integrity of the intestine by the ingestion of two indigestible saccharides that after GI uptake become fully excreted in urine. One of these (usually lactulose) passes the intestinal wall through paracellular transport ('leakage'), while the other (usually mannitol) passes by paracellular and transcellular transport. The urinary lactulose/mannitol ratio is used as a measure of intestinal integrity and permeability (van Elburg et al., 1995). In addition, a recent study found a high prevalence of congenital GI anomalies (adjusted odds ratio 5.1, 95\% confidence interval 1.8-14.1), notably pyloric stenosis, in autism, which may be linked to the high rate of GI dysfunction reported by their parents (Wier, Yoshida, Odouli, Grether, \& Croen, 2006).

The implication of the GI tract in autistic pathophysiology warrants more detailed investigation of the gut-brain axis (Erickson et al., 2005). Especially the role of serotonin (5-hydroxytryptamine; 5-HT), as a messenger within this axis (Gershon, 2005), deserves attention. Many different aspects of the 5-HT system in autism have already been studied (Burgess, Sweeten, McMahon, \& Fujinami, 2006; Croonenberghs, Verkerk, Scharpe, Deboutte, \& Maes, 
2005; Janusonis, 2005; Mulder et al., 2004). A recent report (Mulder et al., 2004) on platelet (PLT) 5-HT in PDD showed PLT hyperserotonemia in approximately $36 \%$ of patients with autism and in 58\% of patients with PDD not otherwise specified (NOS). Using mixture-modeling analysis Mulder et al. (2004) derived an empirical cut-off value that enabled dichotomization of patients with PDD into normo- and hyperserotonemic. Extensive behavioral assessments did, however, not show significant correlates with PLT 5-HT or hyperserotonemic status.

A common (developmental) factor, causing both an autistic brain and deregulated 5-HT release from the GI tract, years after birth, may be involved in the etiology of PDD (Janusonis, 2005). The primary site of the hyperserotonemia in autism is likely to be located in the GI tract. Serotonin is a biogenic amine that derives from the essential amino acid tryptophan (Tryp) by hydroxylation and subsequent decarboxylation. The GI tract contains about $80 \%$ of bodily 5 -HT, which is unevenly distributed among the enterochromaffin cells (90-95\%) and neurons (5-10\%) (Houghton, Atkinson, Whitaker, Whorwell, \& Rimmer, 2003). The main functions of 5-HT are in smooth muscle contraction, blood pressure regulation, and peripheral and central neurotransmission. Serotonin localized in the basolateral stores of enterochromaffin tissue is released upon neuronal, chemical or mechanical stimulation. Several 5-HT receptors control GI motility, sensation and secretion (Gershon, 2005). Following its release, 5-HT is removed from the interstitial space by 5 -HT selective reuptake transporters (Gershon, 2005). However, part of the 5-HT enters the portal blood and systemic circulation where it is either rapidly taken up and accumulated by PLT, or metabolized by the liver, lung and kidneys into its major metabolite 5-hydroxyindoleacetic acid (5-HIAA) (Houghton et al., 2003). Platelets store and transport the majority (99\%) of circulating 5-HT (Ortiz, Artigas, \& Gelpi, 1988).

Elevated PLT 5-HT levels observed in subgroups of patients with PDD, may be related to increased GI motility. This notion is supported by higher PLT 5-HT in patients with diarrhea predominant irritable bowel syndrome (dIBS), as compared with healthy controls (Houghton et al., 2003), although this was not consistently found (Atkinson, Lockhart, Whorwell, Keevil, \& Houghton, 2006). Patients with d-IBS have augmented GI motility, which is likely to cause increased exposure of their circulating PLT to 5-HT (Atkinson et al., 2006; Gershon, 2005; Houghton et al., 2003). Increased PLT 5-HT is also observed in patients with carcinoid tumors (Kema et al., 2001). Carcinoid tumors derive from enterochromaffin cells and are characterized by high 5 -HT production with diarrhea as a frequent symptom (Modlin, Kidd, Latich, Zikusoka, \& Shapiro, 2005). Consequently, measurement of PLT 5-HT is used as a sensitive marker for the early diagnosis and the subsequent follow-up of patients with carcinoid tumors (Kema et al., 2001).

The aim of the present study was to investigate whether the subgroup of children with PDD having increased PLT 5-HT levels, is the same as the one exhibiting increased intestinal permeability as established by a SAT.

\section{Methods}

Patients

Parents of patients with PDD $(n=31)$ according to the DSM-IV TR (American Psychiatric Association, 1994) were asked for the participation of their affected children via the local patient society and pediatricians. Oral and written informed consent were obtained. Information regarding comorbidity, medication, nutritional supplements and the prevalence of GI related complaints was obtained from medical records and with the aid of assisted questionnaires. The study was performed in Curaçao (The Netherlands Antilles) in the summer of 2004. All collected urine and blood samples were transported in dry ice to the Netherlands for further analyses in the University Medical Center Groningen (UMCG). The study was approved by the Medical Ethical Committee of the St. Elisabeth Hospital in Curaçao.

\section{Sugar Absorption Test}

The SAT was performed according to van Elburg et al. (1995). Shortly, the patients ingested a sugar (lactulose, mannitol and sucrose) containing test fluid after an overnight fast. All urine voidings during the following $5 \mathrm{~h}$ were collected and pooled. Urinary sugars were analyzed by gas chromatography as previously described (Jansen, Muskiet, Schierbeek, Berger, \& van der Slik, 1986). A urinary lactulose/mannitol (L/M) ratio above 0.090 was considered to be indicative for abnormal GI integrity/increased intestinal permeability (van Elburg et al., 1995).

\section{Serotonin Assays}

For estimation of 5-HT turnover and exposure of PLT to 5HT, we examined $24 \mathrm{~h}$ urinary excretion of 5-HIAA and total 5-HT (Kema et al., 2001). For this, parents were asked to abstain their child completely from 5-HT containing foods (e.g. banana, pineapple, kiwi, walnuts) during collection and during the preceding $12 \mathrm{~h}$. The volumes of the urine samples were measured before storing at $-20^{\circ} \mathrm{C}$. Urinary 5-HIAA and total 5-HT concentrations were determined as previously reported (Kema et al., 2001). 
Urinary 5-HIAA values were evaluated with the use of agedependent reference values (American Association for Clinical Chemistry, 2005).

Non-fasting venous blood (for serum antibodies) and EDTA-anticoagulated blood (all other assays) were collected from children with PDD. EDTA-anticoagulated blood was placed on melting ice. Hematological indices were measured immediately after sampling. Within $1 \mathrm{~h}$ after collection a 1:1 mixture of $\mathrm{K}_{2}$ EDTA and $\mathrm{Na}_{2} \mathrm{~S}_{2} \mathrm{O}_{5}$ was added to PLT-rich plasma (PRP) to prevent oxidation of indoles. Plasma and serum were stored at $-80^{\circ} \mathrm{C}$. Simultaneous analysis of indoles [Tryp, 5-hydroxytryptophan (5-HTP), 5-HT and 5-HIAA] in PRP was performed as previously described (Kema et al., 2001). PLT 5-HT data were compared with both a local cut-off value of $5.4 \mathrm{nmol} /$ $10^{9}$ PLT (Meijer, Kema, Volmer, Willemse, \& de Vries, 2000) and an empiric cut-off value of $4.55 \mathrm{nmol} / 10^{9} \mathrm{PLT}$ (Mulder et al., 2004). The local cut-off value represents the 97.5th percentile of a reference group of healthy adults, that is employed in our laboratory for the diagnosis of carcinoid tumors. The empirical cut-off value represents the bottom of the valley of the PLT 5-HT bimodal distribution, as exhibited by patients with PDD. This value allows optimal classification into those who are normoserotonemic and hyperserotonemic. Platelet-rich-plasma Tryp data were evaluated with the use of age-dependent reference values (American Association for Clinical Chemistry, 2005).

\section{Exclusion of Celiac Disease}

Serum IgA anti-endomysium titers and HLA genotype were assessed to rule-out celiac disease, which is an established cause of increased GI permeability (van Elburg, Uil, Mulder, \& Heymans, 1993).

\section{Statistics}

All data were analyzed using the Statistical Product and Service Solutions package, version 11.5 (SPSS Inc. Chicago). Data were tested for normality using the ShapiroWilk $W$ test. Group comparisons (normo- and hyperserotonemic) were performed with the Mann-Whitney U test (non-parametric). Spearman (non-parametric) tests were used to evaluate correlations at $\alpha=0.05$, to minimize type-II errors.

\section{Results}

Patients

We enrolled the first 24 (77\%) of the 31 patients with PDD whose parents agreed to participate. Patient characteristics
Table 1 Characteristics of patients with pervasive developmental disorders in Curaçao tested for platelet serotonin and intestinal permeability

\begin{tabular}{ll}
\hline Parameter & \\
Gender (male/female) & $18(75 \%) / 6(25 \%)$ \\
Age (years) & $9.9( \pm 3.9)$ \\
DSM-IV TR diagnoses & \\
$\quad 299.00$ (autistic disorder) & $8(33 \%)$ \\
299.80 (PDD-NOS) & $16(67 \%)$ \\
Ethnicity & \\
$\quad$ Caucasian & $8(33 \%)$ \\
$\quad$ African-American & $13(54 \%)$ \\
$\quad$ Other & $3(13 \%)$ \\
Comorbidity & \\
$\quad$ Epilepsy & $5(22 \%)$ \\
Allergy & $2(9 \%)$ \\
$\quad$ Asthma & $1(4 \%)$ \\
$\quad$ Intestinal yeast infection & $1(4 \%)$ \\
Medication for comorbidity & $9(38 \%)$ \\
Nutritional supplements (vitamins/ $\omega 3$-oils) & $9(38 \%) / 2(4 \%)$ \\
Diet (gluten and casein free) ${ }^{\mathrm{a}}$ & $2(9 \%)$ \\
Physical complaints related to GI tract & $13(57 \%)$ \\
Nausea & $0(0 \%)$ \\
Vomiting & $1(4 \%)$ \\
Diarrhea & $4(17 \%)$ \\
Constipation & $4(17 \%)$ \\
Bloating and gaseousness & $8(35 \%)$ \\
\hline
\end{tabular}

${ }^{\mathrm{a}} n=23$. PDD-NOS pervasive developmental disorder-not otherwise specified

Data represent number (percentage) or mean $( \pm S D)$ for 24 patients, unless otherwise specified

are reported in Table 1. The parents reported 13/23 (57\%) of their affected children to have one or more GI symptoms.

Because of cleanliness problems, no urine samples were obtained from one patient, while from another we only received urine for SAT. Blood sampling from yet another patient was problematic. Consequently, our study comprised urine for SAT from 23/24 patients, $24 \mathrm{~h}$ urine from 22/24 patients and blood samples from 23/24 patients. Table 2 shows the indices related to 5-HT turnover and intestinal permeability of these patients.

\section{Sugar Absorption Test}

Intestinal permeability, reflected by the L/M ratio [median (range) $0.017 \mathrm{~mol} / \mathrm{mol}(0.008-0.035)]$, indicated that none of the patients had increased intestinal permeability (i.e. L/ $M$ ratio $\geq 0.090$ ). 
Table 2 Indices of serotonin (5-HT) turnover and intestinal permeability in patients with pervasive developmental disorders in Curaçao

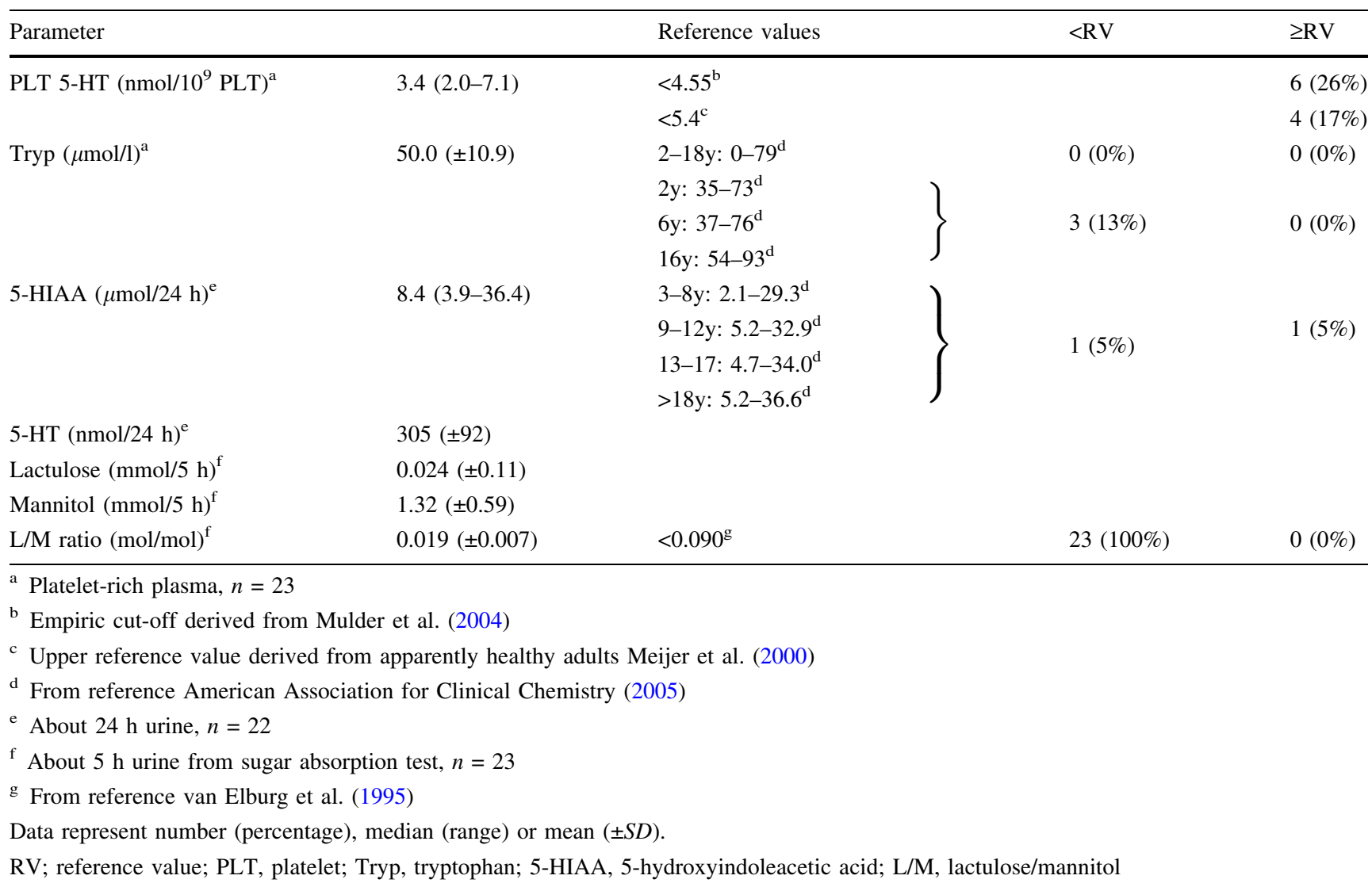

Serotonin Assays

PLT 5-HT [median (range): $\left.3.4(2.0-7.1) \mathrm{nmol} / 10^{9} \mathrm{PLT}\right]$ was elevated in 4 (range: $5.7-7.1 \mathrm{nmol} / 10^{9} \mathrm{PLT}$ ) and 6 [range 4.6-7.1 nmol $/ 10^{9} \mathrm{PLT}$ ] patients if compared to the local cut-off value ((Meijer et al., 2000); $<5.4 \mathrm{nmol} /$ $10^{9}$ PLT) or the empirical cut-off value ((Mulder et al., 2004); $\left.<4.55 \mathrm{nmol} / 10^{9} \mathrm{PLT}\right)$, respectively. The sole patient exhibiting detectable levels of plasma 5-HIAA (26.0 $\mu \mathrm{mol} / 1)$, also exhibited increased PLT 5-HT ( $\left.6.4 \mathrm{nmol} / 10^{9} \mathrm{PLT}\right)$. However, this patient did not exhibit increased urinary total 5-HT or 5-HIAA excretions. Urinary excretion of 5-HIAA was within normal range.

\section{Exclusion of Celiac Disease}

None of the patients were positive for serum $\operatorname{IgA}$ anti endomysium and 8/23 (35\%) patients had a genotype positive for either HLA-DQ2 $(n=5)$ or HLA-DQ8 $(n=3)$. Based on the results of serology, none of the patients seemed to have celiac disease, although we did not perform further tests to exclude this.
Statistics

There was no correlation between PLT 5-HT and L/M ratio ( $p=.663 ; r=-.098)$. Patients exhibiting GI tract complaints did not have higher PLT 5-HT, higher L/M ratios, or higher 5-HIAA and total 5-HT $24 \mathrm{~h}$ urinary excretions $(p>.4)$. Platelet 5-HT correlated with $24 \mathrm{~h}$ urinary 5HIAA excretion $(p=.034 ; r=.465)$. Also the $24 \mathrm{~h}$ excretion rates of 5-HIAA and total 5-HT showed a positive correlation $(p=.005 ; r=.580)$.

\section{Discussion}

In this study of children with PDD we did not observe a relation between PLT 5-HT and intestinal permeability, as derived from the urinary $\mathrm{L} / \mathrm{M}$ ratio. The number of children with PDD exhibiting increased PLT 5-HT was lower compared with reports of others. For instance, the recent study of Mulder et al. (2004) showed 23/81 (28\%) of Dutch children with PDD to exhibit increased PLT 5-HT, using the same analytical method and a cut-off value of $5.4 \mathrm{nmol} / 10^{9} \mathrm{PLT}$. Also our data on intestinal permeability 
contrast with previous reports (D'Eufemia et al., 1996; Horvath et al., 2002) showing that $43-76 \%$ of children with autism have increased intestinal permeability, as established by a SAT. A weakness of the current study is its small size and the lack of a local age- and gender matched control group. We have, on the other hand, no indications for deviant reference values for PLT 5-HT or L/M ratios in Curaçao, as compared with The Netherlands. Age and gender do not appear to affect PLT 5-HT (Mulder et al., 2004), but it must be noted that PLT 5-HT reaches highest levels during childhood and gradually decreases during adulthood (Flachaire et al., 1990). Dependent on the cutoff values employed, we nevertheless found 4 and 6 patients with increased PLT 5-HT. Neither of these patients had abnormal intestinal permeability or L/M ratios residing in the upper range of normality. However, the positive correlation between PLT 5-HT levels and $24 \mathrm{~h}$ urine 5HIAA, and also the relation between urinary 5-HIAA and urinary 5-HT, suggest that, also in PDD, exposure of PLT to 5-HT determines PLT 5-HT and its consistently found increase in a subgroup. It is possible that increased PLT 5HT and GI permeability are not consistent features of children with PDD in time. Long term, e.g. monthly, monitoring of a well defined patient and control group may shed more light on this potential source of variance as a cause of the conflicting results found by several investigators. Differences in the activity of the 5-HT transporter based on genetic polymorphisms are unlikely, since these seem to have minor effects, if any, on PLT 5-HT levels (Mulder, 2006).

In conclusion, the finding of a subgroup of children with PDD exhibiting hyperserotonemia was replicated. None of the children exhibited increased intestinal permeability, while PLT 5-HT was unrelated to both intestinal permeability and GI symptoms. Additional studies are needed to elucidate the etiology of increased PLT 5-HT in PDD and to establish its relation with intestinal pathology, if any.

Acknowledgments We greatly acknowledge Prof. Rainer Bischoff for his valuable contribution to the study design. We also thank the children and their parents for participating in this study and also the many collaborators in the Curaçao health care without whom we would not have been able to conduct these investigations. Furthermore we thank the skilful lab workers of the University Medical Center Groningen (UMCG) for their technical assistance. The work was financially supported by the UMCG and the University of Groningen, The Netherlands. An abstract of this work has been published in the Netherlands Journal for Clinical Chemistry and Laboratory Medicine.

\section{References}

American Association for Clinical Chemistry (2005). Pediatric reference intervals (5th ed.). Washington, DC: AACC Press.
American Psychiatric Association (1994). Diagnostic and statistical manual of mental disorders. Text-Revision (4th ed.). Washington, DC: American Psychiatric Press, Inc.

Atkinson, W., Lockhart, S., Whorwell, P. J., Keevil, B., \& Houghton, L. A. (2006). Altered 5-hydroxytryptamine signaling in patients with constipation- and diarrhea-predominant irritable bowel syndrome. Gastroenterology, 130, 34-43.

Burgess, N. K., Sweeten, T. L., McMahon, W. M., \& Fujinami, R. S. (2006). Hyperserotoninemia and altered immunity in autism. Journal of Autism and Developmental Disorders, 36, 697-704.

Croonenberghs, J., Verkerk, R., Scharpe, S., Deboutte, D., \& Maes, M. (2005). Serotonergic disturbances in autistic disorder: L-5Hydroxytryptophan administration to autistic youngsters increases the blood concentrations of serotonin in patients but not in controls. Life Science, 76, 2171-2183.

D’Eufemia, P., Celli, M., Finocchiaro, R., Pacifico, L., Viozzi, L., Zaccagnini, M., et al. (1996). Abnormal intestinal permeability in children with autism. Acta Paediatrica, 85, 1076-1079.

Erickson, C. A., Stigler, K. A., Corkins, M. R., Posey, D. J., Fitzgerald, J. F., \& McDougle, C. J. (2005). Gastrointestinal factors in autistic disorder: A critical review. Journal of Autism and Developmental Disorders, 35, 713-727.

Flachaire, E., Beney, C., Berthier, A., Salandre, J., Quincy, C., \& Renaud, B. (1990). Determination of reference values for serotonin concentration in platelets of healthy newborns, children, adults, and elderly subjects by HPLC with electrochemical detection. Clinical Chemistry, 36, 2117-2120.

Gershon, M. D. (2005). Nerves, reflexes, and the enteric nervous system: pathogenesis of the irritable bowel syndrome. Journal of Clinical Gastroenterology, 39, S184-S193.

Horvath, K., \& Perman, J. A. (2002). Autism and gastrointestinal symptoms. Current Gastroenterology Reports, 4, 251-258.

Houghton, L. A., Atkinson, W., Whitaker, R. P., Whorwell, P. J., \& Rimmer, M. J. (2003). Increased platelet depleted plasma 5hydroxytryptamine concentration following meal ingestion in symptomatic female subjects with diarrhoea predominant irritable bowel syndrome. Gut, 52, 663-670.

Jansen, G., Muskiet, F. A., Schierbeek, H., Berger, R., \& van der, S. W. (1986). Capillary gas chromatographic profiling of urinary, plasma and erythrocyte sugars and polyols as their trimethylsilyl derivatives, preceded by a simple and rapid prepurification method. Clinica Chimica Acta, 157, 277-293.

Janusonis, S. (2005). Statistical distribution of blood serotonin as a predictor of early autistic brain abnormalities. Theorotical Biology and Medical Modelling, 2, 27.

Kema, I. P., Meijer, W. G., Meiborg, G., Ooms, B., Willemse, P. H., \& de Vries, E. G. (2001). Profiling of tryptophan-related plasma indoles in patients with carcinoid tumors by automated, on-line, solid-phase extraction and HPLC with fluorescence detection. Clinica and Chemica, 47, 1811-1820.

Meijer, W. G., Kema, I. P., Volmer, M., Willemse, P. H., \& de Vries, E. G. (2000). Discriminating capacity of indole markers in the diagnosis of carcinoid tumors. Clinica and Chemica, 46, 15881596.

Modlin, I. M., Kidd, M., Latich, I., Zikusoka, M. N., \& Shapiro, M. D. (2005). Current status of gastrointestinal carcinoids. Gastroenterology, 128, 1717-1751.

Mulder, E. J. (2006). The hyperserotonemia of autism spectrum disorders. University of Groningen. ISBN-13: 978-90-90209593. Retrieved January 26, 2005, from http://dissertations.ub.rug.nl/faculties/medicine/2006/e.j.mulder/?FullItemRecord $=\mathrm{ON}$.

Mulder, E. J., Anderson, G. M., Kema, I. P., de Bildt, A., van Lang, N. D., den Boer, J. A., et al. (2004). Platelet serotonin levels in pervasive developmental disorders and mental retardation: diagnostic group differences, within-group distribution, and 
behavioral correlates. Journal of the American Academy of Child and Adolescent Psychiatry, 43, 491-499.

Ortiz, J., Artigas, F., \& Gelpi, E. (1988). Serotonergic status in human blood. Life Science, 43, 983-990.

van Elburg, R. M., Uil, J. J., Kokke, F. T., Mulder, A. M., van de Broek, W. G., Mulder, C. J., et al. (1995). Repeatability of the sugar-absorption test, using lactulose and mannitol, for measuring intestinal permeability for sugars. Journal of Pediatric Gastroenterology and Nutrition, 20, 184-188. van Elburg, R. M., Uil, J. J., Mulder, C. J., \& Heymans, H. S. (1993). Intestinal permeability in patients with coeliac disease and relatives of patients with coeliac disease. Gut, 34, 354-357.

White, J. F. (2003). Intestinal pathophysiology in autism. Experimental Biology and Medicine (Maywood.), 228, 639-649.

Wier, M. L., Yoshida, C. K., Odouli, R., Grether, J. K., \& Croen, L. A. (2006). Congenital anomalies associated with autism spectrum disorders. Developmental Medicine and Child Neurology, $48,500-507$. 